\title{
AUFSÄTZE
}

\section{Südafrika: Liberal oder demokratisch?}

\author{
Ursula J. van Beek
}

Südafrika ist eine Demokratie, wenn Robert Dahls Konzept der „Polyarchie“1, das Demokratien von Nicht-Demokratien trennt, angewandt wird. Inklusive und gerechte Wahlen mit freiem Wettbewerb sind seit dem Übergang zur Demokratie 1994 regelmäßig im Lande durchgeführt worden, und die Bürger genießen Freiheit der Meinungsäußerung, Zugang zu Alternativinformationen und Verbandsautonomie. Laut einer in der Politikwissenschaft weit akzeptierten Definition kann Südafrika auch als eine Konsolidierungsdemokratie gesehen werden, da Demokratie die einzige Option ${ }^{2}$ zu sein scheint. Es stellt sich allerdings die Frage, ob die politische Praxis innerhalb der demokratischen Institutionen mit den Regeln übereinstimmt, die solche Institutionen benötigen.

Diese Zusammenhänge wurden ausführlich in Bezug auf lateinamerikanische Demokratien diskutiert, wobei die Aufmerksamkeit auf die Dynamik zwischen demokratischer Universalität und den Besonderheiten, die jede menschliche Gesellschaft charakterisieren, gerichtet wurde. Das Argument drehte sich um die Annäherung an - oder die Abweichung von - formalen Regeln. ${ }^{3}$ Obwohl nur schwierig empirisch messbar, ist die Divergenz von formalen Regeln und dem Verhalten politischer Akteure ein Indikator für das Maß demokratischer Qualität. Je kleiner die Abweichung, desto höher die Qualität; je größer die Abweichung, desto schwächer die Qualität und desto größer die Wahrscheinlichkeit, dass nicht-universalistische Beziehungen bestehen. Letzteres schließt gewöhnlich hierarchische Tauschgeschäfte, Patronage und Nepotismus ein, die gemäß den formalen institutionellen Regeln einer Polyarchie als korrupt gelten. Während sich solche Praktiken in den meisten - wenn nicht allen - Polyarchien mehr oder minder finden, und gewiss auch in Südafrika, soll hier etwas anderes im Mittelpunkt stehen: die besondere und spezifisch südafrikanische Interpretation demokratischer Spielregeln durch führende politische Akteure des Landes. Diese Interpretation hat sich die regierende Partei, der Afrikanische Nationalkongress (ANC), bis heute zu eigen gemacht.

\section{Stränge im Befreiungskampf}

Der ANC ist eine altgediente Befreiungsbewegung, die eine Führungsrolle im Kampf gegen die Vorherrschaft der Weißen spielte. Die gebräuchliche Bezeichnung „The struggle“ (der

1 Vgl. Robert A. Dahl, Democracy and its Critics, New Haven 1989.

2 Vgl. Juan J. Linz, Transitions to Democracy, in: Washington Quarterly, 13. Jg. (1990), H. 3, S. $143-163$, S. 156.

3 Vgl. Guillermo A. O'Donnell, Illusions about Consolidation, in: Journal of Democracy, 7. Jg. (1996), H. 2, S. $34-51$. 
Anti-Apartheidskampf) war ein komplexes Gemisch von Konzepten und Aktivitäten, die von den Idealen menschlicher Würde und Gleichberechtigung über gewaltfreie Massenaktionen bis hin zu bewaffnetem Widerstand und Terrorhandlungen gegen den Apartheidstaat reichten. Der Befreiungskampf gründete auf den Werten des Christentums, Liberalismus und Marxismus, des Pan-Afrikanismus und Black Nationalism. All diese Trends bestehen weiterhin, wenngleich in unterschiedlicher Ausdrucksform und in ungleichem Ausmaß, und definieren die gegenwärtige Demokratie des Landes.

Zuerst kam das Ideal. Es wurde von Missionaren verbreitet, den ersten Gegnern der Rassentrennung und einer Segregationspolitik, die sowohl den Kolonialismus als auch die Apartheid untermauerte. Sie standen für Bildung und Christianisierung durch soziale Reformen, die zu einer gleichberechtigten und gerechten Gesellschaft führen sollten. Die Machtbasis dieses gesellschaftlichen Evangeliums waren Missionsschulen und Colleges, die 1953 mit dem Beginn der Säkularisierung verstaatlicht worden waren. Der höchste Ausdruck des christlichen Ideals im politischen Sinne war die 1955 angenommene Freiheitscharta. Das Dokument, das während der Apartheid verboten war, hatte ein gleichberechtigtes und chancengleiches Südafrika im Sinn, das allen gehört, die in ihm leben, mit einem demokratischen Wahlrecht ohne Ansehen von Hautfarbe, Rasse, Geschlecht oder religiöser Überzeugung. Die Freiheitscharta war hauptsächlich von Menschen verfasst worden, die das Bildungssystem der Missionen durchlaufen hatten und, so Beobachter, die biblische Kenntnis aufgesaugt hatten, die ihnen später helfen würde, „die Vision der Unterdrückten

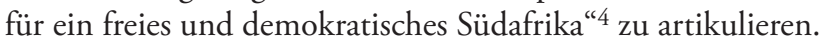

Aber das Christentum kam auf dem Rücken europäischer Kolonisation nach Südafrika und das zu einer Zeit, als anti-christliche Vorurteile sichtbar wurden. Sie hatten den Afrikanischen Nationalismus zur Folge und bereiteten fruchtbaren Boden für den Marxismus. Black Nationalism war eine Rebellion der Jüngeren gegen die Elterngeneration, die in Missionseinrichtungen ausgebildet worden war. Er war darüber hinaus Ausdruck bitterer Ernüchterung vom Christentum als der Religion des scheinheiligen „weißen Mannes“, der das Eine predigte, aber etwas Anderes machte - in besonderer Weise illustriert durch die gesetzlich festgelegte Ungleichheit der Rassen. ${ }^{5}$ Schon vor der Etablierung der Apartheid 1948 entzog die restriktive koloniale Gesetzgebung den meisten Nicht-Weißen ihr Wahlrecht (South Africa Act, 1909), untersagte Schwarzen Landbesitz jenseits kleiner reservierter Bezirke (Natives Land Act, 1913), schuf städtische Segregation durch den Bau von Wohngebieten außerhalb der Ortschaften und Städte, von wo aus Billigarbeiter herangezogen werden konnten (Urban Areas Act, 1923), und schränkte die Bewegungsfreiheit der schwarzen Bevölkerung mithilfe der verhassten Pass Laws - Schwarze mussten außerhalb der ihnen zugeteilten Wohngebiete einen Pass tragen - in der Kapkolonie und in Natal ein. Nach der Etablierung des Rassismus im Jahr 1948 teilte die Gesetzgebung der Apartheid die südafrikanische Bevölkerung in vier ethnische Gruppen: Weiße, Schwarze, Farbige (Südafrikaner gemischter Herkunft) und Inder (Südafrikaner indischer Herkunft). Schwarzen wurde die Staatsbürgerschaft entzogen, Zwangsräumungen und Umsiedelungen ande-

4 C.M. Xundu, The Congress of the People Campaign and the Overall View of the Freedom Charter, in: James A. Polley (Hrsg.), The Freedom Charter and the Future, Kapstadt 1988, S. $13-17$.

5 Die Wortwahl „Rasse” anstelle von „Ethnizität“ ist in Südafrika nicht nur geläufig, sondern durchaus politisch korrekt und wird hier zum besseren Verständnis der kulturellen Hintergründe beibehalten (Anmerkung des Übersetzers). 
rer nicht-weißer Gruppen erlaubt und die Privatsphäre verletzt, indem das Passgesetz flächendeckend eingeführt und Mischehen sowie intime Beziehungen über Rassengrenzen hinweg verboten wurden.

Der Befreiungskampf entstand als Antwort auf den tiefen Eingriff dieser diskriminierenden Gesetze in alle Bereiche des menschlichen Lebens und als Opposition gegen die zunehmend brutalen Methoden, mit denen der Apartheidstaat diese durchsetzte. Im Verlaufe des Befreiungskampfes verschmolzen freiheitliche politische Denkweisen über zukünftige Staatsangehörigkeit in zwei große Strömungen: die „chartistische“ rassenfreie Integration und der Black Nationalism. Im chartistischen Trend koexistierte liberaler Multi-Rassismus, generell verkörpert durch die Freiheitscharta, mit marxistischen Klassenkonzepten in der Vorstellung einer nicht-rassistischen südafrikanischen Gesellschaft nach der Befreiung. Die langwierige Debatte zwischen Marxisten und Black Nationalists drehte sich um die Frage, ob Klasse oder Rasse der richtungsweisende und identitätsprägende Leitsatz der Nation sein sollte. Die Polarisierung in der Denkweise schwarzer Politiker über diese Frage führte 1959 zu einer Abspaltung vom African National Congress (ANC) und der Bildung des Pan Africanist Congress (PAC).

1960 wurde sowohl der ANC als auch der PAC vom Apartheidregime gesetzlich verboten. Es entstanden neue Ideen und Strukturen, nachdem die zwei Hauptorganisationen im Anti-Apartheidskampf in den Untergrund gedrängt und ihre Anführer entweder im Gefängnis oder im Exil waren. Die Black Consciousness Movement (BCM) war die erste Bewegung, die das Vakuum füllte und bis 1976 großen Einfluss ausübte. Ihre rassenorientierte Zukunftsvision beinhaltete nicht nur das Streben nach Befreiung von politischer Unterdrückung wie im Falle des ANC, sondern auch die Befreiung der Psyche schwarzer Menschen; laut der Philosophie des Anführers der BCM, Steven Biko, würden diese nicht ernsthaft den Machtgewinn anstreben, wenn sie zwar an Demokratie, nicht aber an ihren eigenen Wert glaubten. Infolge des berüchtigten Aufstands in Soweto 1976, in den die BCM stark verwickelt war, und in dessen Verlauf hunderte Jugendliche durch die Apartheidpolizei getötet wurden, wurde Biko festgenommen. Als er 1977 in Polizeigewahrsam starb und die BCM verboten wurde, machte sich eine Vielzahl junger Mitglieder der Bewegung die revolutionäre Rhetorik von Karl Marx zueigen in dem Glauben, dass Apartheid nur durch bewaffneten Widerstand zerstört werden könne. Sie strömten aus den Townships der schwarzen Bevölkerung in die vom ANC geleiteten Militärcamps in den Nachbarländern. ${ }^{6}$

Innerhalb des Landes wurde die Leitung des Befreiungskampfes an die United Democratic Front (UDF) übergeben. Obwohl die UDF schon einige Jahre zuvor gegründet worden war, kam sie erst nach dem Aufstand in Soweto 1976 zur vollen Geltung. Der Aufstand, der generell als der Anfang vom Ende der Apartheid betrachtet wird, verbreitete sich bald über alle Townships der Schwarzen und bahnte den Weg für eine politische Kultur, die nicht nur Militanz, sondern auch Massenaktivismus hervorbrachte. Die UDF bildete einen wichtigen nationalen Sammelpunkt für hunderte von anti-Apartheid-orientierten Organisationen der Zivilgesellschaft, die auf lokaler Ebene aus dem Boden schossen. Diese schlossen Kirchen- und Zivilverbände sowie diverse Arbeiter-, Jugend-, Studenten- und Frauengruppen ein. Die UDF war eine rassen- und gewaltfreie Bewegung. Sie brachte

6 Vgl. C.R.D. Halisi, Black Political Thought in the Making of South African Democracy, Bloomington / Indianapolis 1999. 
Menschen aller Rassen jenseits ethnischer und Klassengrenzen zusammen und wurde gemäß der Strategie des zivilen Ungehorsams geformt, wie sie in den 1950er Jahren vom ANC betrieben worden war, mit dem die UDF ein christliches Erbe teilte. Der Antrieb, der bei der Konsolidierung der oppositionellen Front half, war die Bekanntmachung der Regierung, das so genannte Dreikammerparlament (1984 bis 1994) einzurichten. Ziel war es, die politische Macht der regierenden weißen Nationalen Partei weiter abzusichern mit dem Zugeständnis einer eingeschränkten politischen Stimme der farbigen und indischen Bevölkerung Südafrikas, aber unter völligem Ausschluss der schwarzen Bevölkerungsmehrheit vom Wahlprozess. ${ }^{7}$

Die UDF verstärkte und bereicherte den Befreiungskampf in mindestens drei wichtigen Punkten. Da sie mit dem ANC verbündet war und sich in dessen Abwesenheit als Vertreter sah, hielt sie das Image des ANC lebendig und weitete darüber hinaus dessen zukünftige Basis kräftig aus. Dies wurde durch die Aufnahme vieler ländlicher und städtischer Grassroot-Organisationen erreicht. Weiterhin half die UDF als Stellvertreter des ANC, das Gewicht weg von der Black Consciousness Bewegung und hin zu nicht-rassistischen Lösungen zu verschieben; nur einige wenige BCM-Zellen blieben Anfang der 1980er Jahre übrig, wobei allerdings ihr genereller kultureller und ideologischer Einfluss weiterhin besteht. Ausschlaggebend ist hier, dass die UDF nicht nur die Ideale der Freiheitscharta wieder aufleben ließ, sondern diese zur Entwicklung einer Populärdemokratie benutzte, bei der Demokratie nicht nur das Ziel, sondern ebenso der Weg ist: Die Teilnahme am Kampf um Demokratie sollte der Vorbereitung breiter Partizipation in der Demokratie selbst dienen. ${ }^{8}$

Eine kleine, aber hartnäckige Anti-Apartheidstimme im Befreiungskampf kam von einer weißen liberalen parlamentarischen Opposition: Die United Party (UP) und später deren Nachfolgeorganisation, die Progressive Federal Party, plädierten dafür, die Apartheid durch ein föderales System, einen gewaltenteiligen Bundesstaat, zu ersetzen. ${ }^{9}$

\section{Die Entwicklung der Demokratie in Südafrika}

Die liberale Demokratie soll aus zwei in mancher Hinsicht widersprüchlichen Grundelementen bestehen: dem Liberalen und dem Demokratischen. Ersteres bezieht sich auf die Grenzen des Geltungsbereichs und der Reichweite der Regierung im Namen natürlicher Rechte und individueller Freiheit; letzteres betrifft die Souveränität der Mehrheit oder die Herrschaft des Demos ${ }^{10}$, die manchmal durchaus die Garantien des liberalen Konstitutionalismus auszuhöhlen vermag.

7 Vgl. Jeremy Seekings, The UDF: A History of the United Democratic Front in South Africa 19831991, Kapstadt 2000.

8 Vgl. Murphy Morobe, Towards a People's Democracy: The UDF View, in: Review of African Political Economy, 40. Jg. (1987), H. 1, S. 81 - 95.

9 Die zwei prominentesten Abgeordneten waren die Oppositionsführer Fredrik van zyl Slabbert und Helen Suzman, die den aufeinander folgenden Apartheidregimen der National Party erstaunliche 36 Jahre lang Strafpredigten hielten und den wahren Inhalt ihrer unterdrückenden Rassenvorschriften entblößten.

10 Vgl. Fareed Zakaria, The Rise of Illiberal Democracy, in: Foreign Affairs, 76. Jg. (1997), H. 6, S. 22 - 43; Marc F. Plattner, Globalization and Self-Government, in: Journal of Democracy, 13. Jg. (2002), H. 3, S. $54-76$. 
In normativer Hinsicht trug das liberale Element den Sieg davon, als das Ethos der Freiheitscharta im Gründungsdokument des Südafrikas nach der Apartheid zum Ausdruck kam. Der in der Charta bewahrte und Generationen Unterdrückter inspirierende Traum, dass „Südafrika allen gehört, die dort leben“, findet nun seinen Ausdruck in der Präambel der Verfassung, die Anspruch erhebt auf die Errungenschaft eines friedlichen Übergangs zu einer inklusiven, nicht-rassistischen Demokratie, sich bezieht auf die Notwendigkeit, ein vereintes und demokratisches Südafrika zu gestalten, und die bekannt ist für ihre starke Einbürgerung der liberaldemokratischen Werte von Menschenwürde, Gleichheit und Freiheit.

Der liberale Geist der ersten Jahre des demokratischen Südafrika wird in zwei Punkten besonders auffällig: zum einen durch Nelson Mandela, den ersten schwarzen Präsidenten des Landes. Als charismatischer und ungeheuer beliebter Anführer, mit seiner geschätzten Vision von Inklusivität und Versöhnung seines Landes und mit seiner Entschlossenheit, diese Werte in Südafrika und jenseits seiner Grenzen zu fördern, schürte er tiefe Emotionen und säte Hoffnung für einen Neubeginn. Zum anderen war es die Gründung und die Arbeit der Wahrheits- und Versöhnungskommission (TRC). Ihr Vorsitzender war der Friedensnobelpreisträger Erzbischof Desmond Tutu, der die Metapher der „Regenbogennation" prägte, die das erhoffte Ideal aller Südafrikaner - Einheit in Vielfalt - zusammenfasste. ${ }^{11}$

Doch der liberale Geist der Demokratie verblasste während der Amtszeit des Präsidenten Thabo Mbeki, dem Nachfolger Nelson Mandelas. Unter seiner Führung transformierte sich der ANC zu einer avantgardistischen Partei, die anscheinend ganz allein wusste, was das Beste für das Volk war. Von einer elitären Führung wurde der Andersgesinnte immer mehr zum Schweigen gebracht und der lebhafte liberale Geist der Beteiligungsdemokratie, der die Periode der UDF und die frühen Jahre nach dem Übergang gekennzeichnet hatte, größtenteils vernichtet. Aus dem Exil zurückgekehrte ANC-Mitglieder, einschließlich $M_{b e k i}{ }^{12}$, brachten ein Kulturverständnis mit, das sich seit über dreißig Jahren außerhalb des Landes entwickelt hatte, wo die meisten Ausgewanderten unter Bedingungen geheimer Operationen und in Angst vor Infiltration, Angriffen oder Ermordung gelebt hatten. Diese durch westliches Misstrauen verstärkten Lebensumstände und Erfahrungen schweißten sie zu einer hochzentralisierten hierarchischen Gruppe zusammen, die stark von sowjetischen und ostdeutschen Verbündeten sowohl beeinflusst wurde, als auch auf diese angewiesen war. Die Exilpolitiker waren es auch, die das Konzept des Uhuru African Nationalism der 1960er Jahre, gemischt mit dem 1970er Black Nationalism oder Afrikanismus von Steven Biko wieder mitbrachten, das durch die ernüchterten Mitglieder des BCM ins Exil getragen worden war. Die vielfältigen Elemente der Exilkultur vermischten sich und wirkten sich zunehmend auf das Südafrika nach Mandela aus. Das Kulturverständnis wandelte sich in Afrikanismus und demokratischen Zentralismus um, mit hinter verschlossenen Türen aus-

11 Zur Arbeit der TRC vgl. Ursula J. van Beek, Reflections on Reconciliation: The Case of South Africa, in: Björn Hettne (Hrsg.), Culture, Security and Sustainable Social Development, Houndmills u.a. 2008.

12 Anders als die Mehrheit der ANC-Mitglieder, die ins Exil oder in andere afrikanische Länder gingen, verbrachte Thabo Mbeki einige seiner Exiljahre in der Sowjetunion, aber auch in England, wo er sich zur Sozialdemokratie und den Reformen des „Third Way“ der britischen Labour Party hingezogen fühlte. Die Wirtschaftspolitik seiner Regierung lehnte sich offenbar an letztere an. 
gearbeiteten Politiken, die zur gehorsamen Ausführung gemäß Rangordnung heruntergereicht werden ${ }^{13}$, obwohl die liberalen Institutionen der Demokratie formal etabliert blieben.

\subsection{ANC-Alleinregierung: Abkehr vom Prinzip des Liberalismus}

Bei der 50. Nationalkonferenz des ANC $1997^{14}$ in Mafikeng wurde das Konzept der „Afrikanischen Hegemonie im Kontext einer multikulturellen und rassefreien Gesellschaft" formuliert; seitdem ist das nationale Ziel nicht eine liberaldemokratische Gesellschaft Gleichberechtigter, sondern die „Nationaldemokratische Revolution“ (NDR), die die Lebensqualität besonders der Armen, die per definitionem hauptsächlich schwarz sind, verbessern soll. Mbekis politische Reden machten klar, dass nicht die Gleichberechtigung der Individuen, sondern der „nationalen Gruppen“, rassisch ausbalanciert „throughout all its sectors, classes and status orders would form the normative basis of the South African society" 15 . Damit wurde vom liberalen Prinzip gleicher Individualrechte abgewichen. Gemäß dem Umgestaltungsparadigma der „Gleichberechtigung zwischen nationalen Gruppen“ kann ein südafrikanischer Bürger nun nicht mehr als gleichberechtigtes und autonomes Individuum nach seinem eigenen Besten innerhalb eines insoweit neutralen Staates streben, sondern als zugeordnetes Mitglied einer bestimmten (sub-)nationalen Gruppe, der der Staat zuvor eine bestimmte Auffassung von Gemeinwohl vorgegeben hat. Dies widerspricht dem Konzept von Gleichheit, wie es in der Verfassung steht, die die Bürger Südafrikas angenommen haben. ${ }^{16}$

Dieser normative Politikwechsel schränkt zwar nicht die fundamentalen Menschenbeziehungsweise Bürgerrechte der Meinungs- und Gedankenfreiheit, der Freiheit, sich zusammenzuschließen und sich politisch zu beteiligen, ein; sehr wohl ist damit aber bewirkt worden, dass die Unabhängigkeit Einiger zur Förderung des Gemeinwohls Anderer eingeschränkt wird. Die Rechte einer Gruppe über die Rechte anderer Gruppen läuft aber den Idealen einer gerechten Gesellschaft zuwider. Prinzipiell ließe sich durchaus argumentieren, dass eine Abweichung von „den Spielregeln“ gerechtfertigt ist, um den Ungerechtigkeiten der Vergangenheit Abhilfe zu schaffen. Doch in der Praxis hat diese Politik die südafrikanische Demokratie weitaus mehr geschwächt als gestärkt.

Innerhalb des neuen Transformationsparadigmas und Mbekis international geförderter Philosophie der Afrika-Renaissance fand der wiederauflebende Afrikanismus eine Nische. Das Ergebnis war die Entstehung eines exklusiven Kulturnationalismus auf Kosten von inklusivem zivilen Patriotismus. Damit wurde den anderen „nationalen Gruppen“ in der südafrikanischen Gesellschaft zusätzlich zu der Tatsache, dass sie wirtschaftlich benachteiligt

13 Vgl. J. Daniel, The Mbeki Presidency: Lusaka Wins, in: South African Institute of International Affairs (Hrsg.), South African Yearbook of International Affairs 2001/2002, Johannesburg 2002.

14 African National Congress, Strategy and Tactics. As amended at the 50th National Conference 1997, http://www.anc.org.za/ancdocs/history/conf/conference50/strategyamend.html (Abruf am 25. Mai 2010).

15 Thabo Mbeki, Africa - The Time Has Come: Selected Speeches, Kapstadt 1998.

16 Eine detaillierte Analyse liefert Peter Hudson, Liberalism, Democracy and Transformation in South Africa, in: Politikon, 27. Jg. (2000), H. 1, S. $93-102$. 
sind, das Gefühl kultureller Entfremdung und politischer Machtlosigkeit vermittelt. ${ }^{17}$ In der Summe kam es zu einer Verletzung liberaldemokratischer Normen inklusiver Gleichberechtigung und damit praktisch zu einer Abkehr von Nelson Mandelas Vorstellung von „einer Nation mit einem gemeinsamen Schicksal“, wo Menschen das Gute aus ihrer jeweiligen historischen Vergangenheit nehmen und ihre Zukunft gemeinsam gestalten. ${ }^{18}$ Wie Ernest Renan im 19. Jahrhundert bemerkte, ist es eben dieses „Verlangen, dauerhaft zusammen zu leben“, das die Basis einer demokratischen Gesellschaft ausmacht; ohne dies besteht große Gefahr, denn es kann keine Demokratie ohne einen vereinten demokratischen Demos geben.

Die Abkehr von inklusiver Staatsbürgerschaft und die Aufteilung der südafrikanischen Nation in „Gruppen“ ging mit einem zunehmend spaltenden und arroganten Führungsstil einher. Als Mbeki wiederholt den Willen des Volkes missachtete, wirkte sich dies auf die gesamte Gesellschaft aus. Entgegen medizinischer Beweise und der weit verbreiteten Auffassung des Volkes und trotz vieler Aufrufe zivilgesellschaftlicher Organisationen änderte der Präsident niemals seine negierende Haltung gegenüber der AIDS-Pandemie. Auf dieser Grundlage stellten er und seine loyale Gesundheitsministerin den kostenlosen Zugang zur Behandlung von AIDS zurück - mit katastrophalen Konsequenzen für die Erkrankten. ${ }^{19}$ Mbeki beharrte auch auf seiner "stillen Diplomatie“ mit Simbabwe, obwohl nicht nur die internationale Gemeinschaft, sondern auch die südafrikanischen Gewerkschaften auf einen aktiveren Ansatz drängten, da sie mit den simbabwischen Arbeitern sympathisierten. Viele Südafrikaner beschuldigten ihr Staatsoberhaupt, mehr mit der Besänftigung Robert Mugabes beschäftigt zu sein, als mit den zahlreichen Problemen, die durch die ins Land strömenden Simbabwer entstehen. ${ }^{20}$

Bei innenpolitischen Fragen zog Mbeki sein Kabinett, oder genauer, seinen loyalen inneren Kreis, von Gesprächen und Verhandlungen mit allen anderen Akteuren zurück, einschließlich der Opposition. Außerdem verschob sich während seiner Amtszeit die Balance zwischen Exekutive und Legislative erheblich, da er etliche Zuständigkeiten und Funktionen an sich zog beziehungsweise in seinem Präsidialbüro bündelte, so dass das Parlament an politischem Einfluss verlor. Noch gravierender ist der Vorwurf, dass Mbeki daran beteiligt war, als die ANC-Führung versuchte, die parlamentarische Untersuchung angeblicher Korruptionsfälle in Südafrikas bisher größtem Waffenhandel zu blockieren. ${ }^{21}$ Viele Kommentatoren in Südafrika stimmen heute in der Auffassung überein, dass: viel von dem, was im Lande

17 Die gesetzliche Durchsetzung von Affirmative Action (Förderung von Minderheiten) und Black Economic Empowerment (BEE), der ökonomische Bemächtigung schwarzer Südafrikaner, erteilt Erwerbstätigkeitsvorzüge anhand eines auf Rasse (ethnischer Gruppe) basierenden Quotasystems, beschleunigt Beförderungen und Positionen in Managementbereichen und zwingt Betriebe, Firmen und Gesellschaften eine Anzahl schwarzer Partner und Vorstandsmitglieder zu ernennen.

18 Nelson Mandela, Mandela eer Afrikaners by monument, in: Die Burger vom März 2002.

19 Forscher der Harvard Universität glauben, dass die AIDS-Politik der Regierung Mbekis direkte Verantwortung für den vermeidbaren Tod von ungefähr 300.000 Menschen im Land trägt. Vgl. Sarah Boseley, Mbeki Aids Denial 'Caused 300,000 Deaths', in: The Guardian vom 26. November 2008, http://www.guardian.co.uk/world/2008/nov/26/aids-south-africa (Abruf am 25. Mai 2010).

20 Vgl. Tererai Karimakwenda, South Africa: Mbeki Blamed After 20 More Die in Xenophobic Attacks, in: SW Radio Africa vom 19. Mai 2008, http://allafrica.com/stories/200805191523.html (Abruf am 25. Mai 2010).

21 Vgl. Andrew Feinstein, After The Party. A Personal and Political Journey inside the ANC, Johannesburg 2009. 
„verkehrt läuft, besonders der brutale Nachfolgekrieg im ANC und der stets wachsende Nexus zwischen Wirtschaft und Politik, seine Wurzeln im Waffenhandel [hat]"22.

Was letztendlich den politischen Untergang Mbekis besiegelte, war seine Geringschätzung der Positionen und Erwartungen der Gewerkschaft COSATU und der Kommunistischen Partei (SACP), Partner des ANC in der regierenden Dreier-Allianz. Dabei ist hinzuzufügen, dass weder die SACP noch COSATU als eigenständige Organisationen ins Parlament eingezogen sind, sondern unter dem Dach des ANC antraten. Alle ihre Abgeordneten sind gleichzeitig Mitglieder des ANC. Die Kombination von Mbekis Führungsstil und seiner pro-kapitalistischen Wirtschaftspolitik setzte ihn auf einen Kollisionskurs mit einem Großteil der Wählerschaft und den linken Mitgliedern der regierenden Allianz. Der interne Unfriede verstärkte sich weiter im Laufe des Jahres 2005, als Mbeki seinen Stellvertreter Jacob Zuma wegen mehrerer Korruptionsverfahren entließ. In den folgenden zwei Jahren wurden die internen Machtkämpfe im ANC immer heftiger, und der in der Linken verankerte Zuma zeichnete sich als der Mann des Volkes ab, der im Gegensatz zum unnahbaren Mbeki zuhörte. Zumas Name wurde zum Symbol von Widerstand und Kampf gegen Ungerechtigkeit. Die internen Auseinandersetzungen wurden durch ein Misstrauensvotum entschieden, das der ANC-Vorstand auf der 52. Nationalkonferenz der Partei im Dezember 2007 in Polokwane einbrachte. Mbeki wurde von seinen Pflichten als Präsident des ANC und später als Staatsoberhaupt entbunden, während Jacob Zuma der neue Präsident des ANC wurde und in den darauf folgenden Wahlen im April 2009 auch das Amt des Präsidenten von Südafrika antrat. ${ }^{23}$

\subsection{Der Parteitag von Polokwane 2007 als Wendepunkt}

Die Konferenz in Polokwane markierte einen Wendepunkt und war ein deutlicher Sieg der Linken. Er rekonfigurierte die Führungselite und verlagerte die Rhetorik vom Black Nationalism der Administration Mbekis schärfer zur Linksfraktion des ANC, eng verbündet mit den Gewerkschaften und der Kommunistischen Partei. Die in Polokwane gefassten Beschlüsse bestätigten wieder den Ehrgeiz des ANC „[to remain] the key strategic centre of power, which must exercise leadership over the state and society in pursuit of the objectives of the NDR ... [and] make the decisions on the direction our country should take collectively" ${ }^{24}$. An die marxistisch-leninistische Sprache der ehemaligen Sowjetunion und des gesamten Ostblocks erinnernd, zeigte sich hier deutlich die Angst des ANC, oder „der Bewegung“, vor einer Gegenrevolution: „The movement should ... exercise vigilance against forces which seek to take advantage of divisions within the democratic movement, in order to subvert and disrupt the agenda of revolutionary change. In this regard, it should be noted that such threats as organized crime and susceptibility of the lumpen-proletariat to recruitment by reactionary forces can have counter-revolutionary implications."

22 Mail \& Guardian Online vom Januar 2007, http://www.mg.co.za/ (Abruf am 25. Mai 2010).

23 Vgl. Claire Ceruti, African National Congress Change in Leadership: What Really Won it for Zuma, in: Review of African Political Economy, 35. Jg. (2008), H. 115, S. 107 - 114.

24 Alle nachfolgend zitierten englischsprachigen Auszüge sind Dokumenten entnommen, die die Beschlüsse der Konferenz in Polokwane enthalten, http://www.anc.org.za/ancdocs/history/conf/ conference52/ (Abruf am 25. Mai 2010). 
Das Gesellschaftskonzept der nationalen Gruppen beibehaltend wurden die weißen Südafrikaner, die zuvor alle in der gegenrevolutionären Kategorie zusammengeworfen worden waren, in Polokwane als aus mehreren Sektionen bestehend kategorisiert, und diese müssten behandelt werden ,in the context of the responsibility of the ANC to mobilise all South Africans behind the project of social transformation”. Doch die Hauptaufgabe des ANC sei und bliebe es, „to mobilise all the classes and strata that objectively stand to gain from the success of the cause of social change”. Die Partei wurde aufgerufen, die „Massen zu erziehen": „The fact that a particular group, class or stratum stands to benefit form such transformation does not necessarily mean that it will automatically be aware of it. Thus, the task of education, organisation and mobilisation is critical at all stages. This is as important in this period as it was in the past; for, in as much as the people were their own liberators, success today is contingent upon transformation being people-centred and people-driven."

Wird demokratische Transformation so verstanden, werden nur jene einbezogen, die sich mit den „Imperativen” der Verfassung korrekt identifizieren, das heißt diese in Übereinstimmung mit der durch den ANC konstruierten Nationaldemokratischen Revolution interpretieren. Diese lässt keinen Raum für anderweitige Richtungen der Politik außerhalb der Auslegung des ANC und negiert die Pluralität unterschiedlicher Sichtweisen, eines der grundlegenden Konzepte liberaler Demokratie. Entweder unterstützt man in Südafrika Zielvorstellungen der demokratischen Revolution, oder man ist ein Gegenrevolutionär. Erstere identifizieren sich mit „der Perspektive und den Werten des ANC (Entwicklungsnation, Kollektivrechte, soziale Werte und Gemeinschaftssolidarität, Ubuntu ${ }^{25}$, Nicht-Sexismus, usw.)“. „Gegenrevolutionäre“ folgen aus Sicht des ANC der ideologischen Perspektive der Medien und stehen für Werte wie Neoliberalismus, schwache und passive Nation, für eine Überbetonung der Rechte des Einzelnen, Wirtschaftsfundamentalismus usw. Die Grenzlinie wurde durch den „Ideenkampf” definiert und wie folgt beschrieben: „A major ideological offensive, largely driven by the opposition and fractions in the mainstream media, whose key objective is the promotion of market fundamentalism, control of the media and the images it creates of a new democratic dispensation in order to retain old apartheid economic and social relations."

Die Medien sollten, laut der Beschlüsse der Konferenz, zu der Transformation des Landes beitragen, indem sie die als Grundsatz der Nationaldemokratischen Revolution spezifizierten Zielvorstellungen unterstützen, anstatt Spiegelbild der „ideologischen Kämpfe und der auf Rasse, Klasse und Geschlecht basierten Machtbeziehungen in unserer Gesellschaft” zu sein. Im „Ideenkampf“ geht es allerdings nicht so sehr um die überwölbenden Konzepte von Rasse, Klasse oder Geschlecht, sondern eigentlich um das Individuum und seine Rechte. Die als voreingenommen beschriebenen Medien sind einige wenige gedruckte, die die Meinungs- und Pressefreiheit im libertären Sinne wahrnehmen. Das Ziel ihrer Redaktionspolitik ist es, das Gemeinwohl aller Bürger und nicht lediglich das Wohl Einiger zu schützen. Diese Medien fungieren als Kontrolleure gegen die Regierungsmacht, eine Funktion,

25 Ubuntu bedeutet wörtlich aus Zulu übertragen: „Ein Mensch ist ein Mensch durch andere Menschen”. In der afrikanischen Philosophie ist Ubuntu ein Konzept, das auf Respekt und Mitgefühl für andere beruht; es kombiniert Regeln des sozialen und ethischen Verhaltens mit traditionellem religiösen Glauben der Vorfahren und verheißt jenen, die zu ihren Lebzeiten die Prinzipien des Ubuntu einhalten, dass sie selbst verehrte Vorfahren werden und im Tode Einheit mit den Lebenden erreichen. 
die sich in einem scharfen Konflikt mit der Agenda einer Regierung befindet, die sich selbst als „das strategische Machtzentrum [sieht], das Führung über den Staat und die Gesellschaft ausüben muss".

Einer der Beschlüsse der Konferenz mit dem Titel "Strategie und Taktiken des ANC“ beschrieb Südafrika als ein „System einer dynamischen Mehrparteiendemokratie“. Die Aussage trifft insoweit zu, als eine Vielzahl unterschiedlicher Parteien existiert und es ihnen frei steht, am politischen Wettbewerb und den Wahlen teilzunehmen. Doch die Dynamik des Systems muss noch getestet werden, denn bisher gab es keine ernst zu nehmenden Rivalen für den ANC. Fest steht, dass die Fähigkeit des ANC, die Nationaldemokratische Revolution zu gestalten und umzusetzen, darauf gründet, dass sie bei allen Wahlen seit dem Übergang zur Demokratie im Jahr 1994 eine solide Mehrheit erhalten hat.

Dies ist der Fall trotz des gegenwärtigen Wahlsystems, das entwickelt wurde, um dieser seinerzeit antizipierten überwältigenden Stärke des ANC entgegenzuwirken. Da das traditionelle Mehrheitswahlsystem anderen Parteien keine angemessene Repräsentation gesichert hätte, entschied man sich für ein Verhältniswahlrecht. Die anhaltend große Mehrheit des ANC wurde so aber nicht verhindert; vielmehr wirkte sich das Verhältniswahlsystem negativ auf die Repräsentationsbeziehungen zwischen Wählern und Gewählten aus. Deren Verbindung war bisher bestenfalls dürftig, zum Teil gar nicht existent, denn obwohl Abgeordneten informell bestimmte Wahlbezirke zugeordnet wurden, die sie vertreten sollen, hängt ihre Wiederwahl nicht von den Bürgern dieser Wahlbezirke, sondern von ihrem Platz auf der durch den ANC erstellten Liste ab. Der ANC übersieht die allgemein anerkannte Tatsache, dass eine Wahlreform zur Erhöhung parlamentarischer Rechenschaftspflicht momentan die größte Herausforderung der Politikgestaltung ist ${ }^{26}$, und entschied auf der Konferenz in Polokwane, dass „das gegenwärtige Wahlsystem beibehalten und gestärkt werden sollte, um die Verbindung zwischen dem Volk und seinen öffentlichen Vertretern weiter zu vertiefen".

\subsection{Die Parlamentswahl vom 22. April 2009: erhoffter Wandel bleibt aus}

Diese Sichtweise wurde durch zwei politische Akteure, die vor der Wahl 2009 auf oppositioneller Seite Fuß gefasst hatten, einer Prüfung unterzogen. Zum einen war dies der Congress of the People (COPE), eine Neugründung, zu der sich ANC-Dissidenten seit der Konferenz in Polokwane zusammengeschlossen hatten. In seinem Wahlprogramm $2009^{27}$ rief COPE zu einer Direktwahl des Präsidenten, der Premierminister und Bürgermeister auf, um die Rechenschaftspflicht zu erhöhen und die Teilnahme der Öffentlichkeit auf allen politischen Ebenen zu verbessern. Die neue Partei versprach den Bürgern, dass sie künftig ihre öffentlichen Vertreter im nationalen und in den regionalen Parlamenten in einer Kombination aus Direktwahl und Verhältniswahl wählen könnten.

Der zweite Akteur war die Demokratische Allianz (DA), ein politischer Veteran, der seine Wählerschaft langsam, aber sicher ausgebaut hatte. Die DA ist eine Partei liberaler Tradition,

26 Vgl. Dren Nupen, Elections, Constitutionalism and Political Stability in South Africa, in: African Journal on Conflict Resolution, 4. Jg. (2004), H. 2, S. 119 - 143.

27 Vgl. COPE, A New Agenda 2009. Election Manifesto, http://www.congressofthepeople.org.za/ (Abruf am 25. Mai 2010). 
die ihre Wurzeln in der anti-Apartheid-gesinnten Progressive Federal Party hat. ${ }^{28}$ Der Slogan ihrer Wahlkampagne 2009 lautete: „Open Opportunity for All“; er sollte im Kontrast stehen zu dem, was die DA als die von der ANC-Regierung verfolgte „closed patronage society for some“ sah. Bei einem Medienbriefing im Parlament im Januar 2009 legte die Parteivorsitzende Helen Zille ${ }^{29}$ einen Vorschlag zur Änderung des Wahlrechts vor. Sie rief, wie schon COPE, zu einer Direktwahl des Präsidenten, der Premierminister und Bürgermeister auf und präsentierte einen detaillierten Plan für gemischte Verhältniswahlen, um eine direkte Verbindung zwischen Wählern und Wahlkreisabgeordneten herzustellen, der die Rechenschaftspflicht erhöhen würde, ohne das Prinzip der Verhältnismäßigkeit zu zerstören.

Die liberale Gesinnung der DA hat sich über die Zeit bewährt; COPE blieb hingegen eine unbekanntere Größe. Einige seiner Kritiker nannten ihn die „Schwarze DA“, der Vorsitzende selbst bezeichnete seine Partei als „den echten ANC“, und Zyniker titulierten ihn als das politische Zuhause loyaler Anhänger Mbekis. Die neue Partei versprach der Wählerschaft ein vereintes, nicht-rassistisches und nicht-sexistisches Südafrika und erinnerte damit stark an die Ideale der UDF. Sie nominierte einen methodistischen Bischof als Kandidaten für die Präsidentschaftswahl im April 2009 - ein offensichtlicher Versuch, den Glauben unter Südafrikanern, die korrupten Politikern überdrüssig sind, an eine ehrliche und vertrauenswürdige Führung wieder herzustellen,

Wohl als Reaktion auf die Gründung von COPE organisierte der ANC seinen bisher stärksten Wahlkampf. Zuma versuchte, ein kuscheliges Image in seiner Partei zu vermitteln und drängte die Wähler, den Herd des ANC nicht zu verlassen, wenn sie nicht „draußen im Regen stehen wollten“. Schließlich schafften es weder COPE noch die DA, den riesigen ANC zu entthronen. Der Sieg des ANC stand nie in Frage, doch die meisten Kommentatoren hatten erwartet, dass die weit verbreitete Unzufriedenheit der Wähler mit dürftigen Dienstleistungen in einer stark reduzierten Mehrheit resultieren würde. Dies war am 22. April 2009 nicht der Fall, wenngleich der Stimmenanteil des ANC sank - von 69,7 Prozent im Jahr 2004 auf 65,9 Prozent, also knapp an einer Zweidrittelmehrheit vorbei. Die regierende Partei bewahrte ihre dominierende Position, wie die folgende Tabelle zeigt.

Neben der DA und COPE erzielten die übrigen Parteien gemeinsam nur gut zehn Prozent der Stimmen. Nur 13 der 26 Parteien, die an der Wahl teilgenommen hatten, gewannen Mandate in der aus 400 Sitzen bestehenden Kammer. Der militaristische und den Black Nationalism vertretende Pan-Africanist Congress (PAC), ein Hauptakteur des Befreiungskampfes, erhielt lediglich einen einzigen Sitz und besteht nahezu nicht mehr. Die ehemals starke ethnisch fundierte Zulu Inkatha Freiheitspartei (IFP), die in den Wahlen von 1994 von mehr als zehn Prozent der Wählerschaft unterstützt wurde, verzeichnete eine weiter abnehmende Beliebtheit und fiel von 28 Mandaten auf nur 18. Die schwache Leistung der Partei wurde größtenteils dem so genannten Zuma-Faktor zugeschrieben. Es erwies sich, dass der charismatische Jacob Zuma, selbst ein Zulu, eine stärkere Anziehungs-

281989 schloss sich die Progressive Federal Party mit zwei kleineren liberalen Parteien, dem National Democratic Movement und der Independent Party, zur Democratic Party zusammen. Diese vereinte sich im Jahr 2000 mit der New National Party und der Federal Alliance zur Democratic Alliance (DA).

29 Helen Zille war zu jener Zeit Bürgermeisterin von Kapstadt und wurde 2008 zur Weltbürgermeisterin gewählt. Zu ihrem Wahländerungsantrag vgl. Mail \& Guardian Online, Democratic Alliance Proposals for Electoral System Are a Cut Above, 22. Januar 2009, http://www.thoughtleader. co.za/tag/accountability (Abruf am 25. Mai 2010). 
Tabelle 1: Ergebnisse der Wabl zur südafrikanischen Nationalversammlung am 22. April 2009

\begin{tabular}{|l|r|r|r|}
\hline & Stimmen & Prozent & Mandate \\
\hline registrierte Wähler & 23.180 .000 & & \\
gültige Stimmen & 17.680 .729 & & \\
Wahlbeteiligung & & 77,30 & \\
\hline African National Congress & 11.650 .748 & 65,90 & 264 \\
Democratic Alliance & 2.945 .829 & 16,66 & 67 \\
Congress of the People & 1.311 .027 & 7,42 & 30 \\
African Christian Democratic Party & 142.658 & 0,81 & 3 \\
African People's Convention & 35.867 & 0,20 & 1 \\
Azanan People's Organisation & 38.245 & 0,22 & 1 \\
Independent Democrats & 162.915 & 0,92 & 4 \\
Inkatha Freedom Party & 804.260 & 4,55 & 18 \\
Minority Front & 43.474 & 0,25 & 1 \\
Pan-African Congress & 48.530 & 0,27 & 1 \\
United Christian Democratic Party & 66.086 & 0,37 & 2 \\
United Democratic Movement & 149.680 & 0,85 & 4 \\
Vryheidsfront Plus & 146.796 & 0,83 & 4 \\
\hline Anmerkung: Hier sind nur jene Parteien verzeichnet, die mindestens einen Sitz gewonnen haben. \\
Quelle: Official South African Election Results 2009, Independent Electoral Commission (IEC). \\
\hline
\end{tabular}

kraft auf Zulu-Stolz und -Nationalismus ausübte als der alternde und wenig inspirierende Vorsitzende der IFP, Mangosuthu Buthelezi.

Der „Zuma-Faktor“ hat das politische Feld auch in weiterer Hinsicht verändert. Er hat die so genannte Xhosa Nostra beendet, die durch die Xhosas dominierte Führung des ANC. Die Xhosas sind eine ethnische Gruppe, zu der sowohl Nelson Mandela als auch Thabo Mbeki gehören. Die neue Zusammensetzung signalisiert möglicherweise eine Abnahme der ethnischen Spannungen, die schon in der Vergangenheit, aber besonders im Vorfeld der ersten demokratischen Wahlen 1994 eine ernsthafte Bedrohung für die Demokratie in Südafrika darstellten.

Während sich die ethnischen Gewichte in der Wahl 2009 ein wenig verschoben, blieb die Rassenorientierung größtenteils unverändert. Allerdings ist Rasse keineswegs der einzige bestimmende Faktor des Wählerverhaltens. Nimmt man Klasse, Einkommen oder ländlicher versus städtischer Wohnsitz der Wähler hinzu, wird das Bild sehr viel deutlicher und zeigt, dass der ANC besonders in ländlichen Gebieten und von der schwarzen Bevölkerung mit niedrigerem Einkommen unterstützt wird. Angesichts der Geschichte und Demographie Südafrikas und der Tatsache, dass alle großen Parteien des Landes vorgeben, inklusiv zu sein, und um Wähler aller Rassen und Ethnien konkurrieren, kommt es aber vor allem darauf an, wo diese ihre Interessen am besten aufgehoben sehen - und die Auffassung ist, dass der ANC sich am besten um die schwarze Mehrheit kümmert, was die Minderheitsgruppen nach einem anderen politischen Zuhause suchen lässt. Dieser Trend wurde besonders deutlich in der Provinz Western Cape, wo, mit Ausnahme der Inder, der Großteil der Minderheiten lebt und wo schwarze Wähler traditionell den ANC unterstützen, wohingegen die weißen und gemischten Minderheiten oppositionelle Parteien, hauptsächlich die DA, wählen. So erhielt der ANC 2009 dort 31,55 und die DA 51,46 Prozent der Stimmen. 
Das Auftreten von COPE schürte im Vorfeld der Wahlen hohe Erwartungen an ein ReAlignment in Südafrika. Doch die neue Partei trat relativ spät in den Wahlkampf ein und schaffte es aufgrund fehlender Hilfsmittel und schwacher Organisation nicht, ihre Botschaft besonders an die arme schwarze Wählerschaft zu vermitteln. Nach den Wahlen verschwand sie weitgehend. Es gelang aber COPE mehr als irgendeiner anderen Partei, Unterstützung von Wählern aus dem gesamten politischen Spektrum zu bekommen. Hieraus lässt sich ableiten, dass - sollte eine überlebensfähige Opposition zum ANC entstehen - ein inklusives Programm anstelle eines exklusiven Rassenbildes der entscheidende Faktor für den Wahlerfolg sein könnte. So setzte sich ausweislich einer repräsentativen Umfrage die Wählerschaft von COPE aus circa 60 Prozent Schwarzen und je circa 20 Prozent Weißen und Gemischt-Rassigen zusammen. Hingegen waren 96 Prozent der ANC-Wähler Schwarze, während die Unterstützung für die DA zu knapp zwei Dritteln von Weißen und nur zu sechs Prozent von Schwarzen kam. ${ }^{30}$

Die Wahlen 2009 waren die vierten demokratischen in Südafrika seit April 1994. In den Wahlen 1994 erhielt der ANC 62,6 Prozent der Stimmen, sicherte sich 252 der 400 Mandate in der Nationalversammlung und gewann sieben der neun Provinzwahlen. Nur in zwei Provinzen kam es zu anderen Regierungsmehrheiten: in Western Cape die National Party und die Inkatha Freedom Party in Kwazulu-Natal. Insgesamt wählten mehr als 19,5 Millionen Menschen, denn eine liberale Praxis räumte sowohl Staatsbürgern als auch Ausländern mit unbefristeter Aufenthaltsgenehmigung das Stimmrecht ein. Am 9. Mai 1994 wählte die Nationalversammlung einstimmig Nelson Mandela zum Präsidenten von Südafrika. $^{31}$

Bei der zweiten Wahl zur Nationalversammlung im Juni 1999 war das Stimmrecht auf Staatsbürger ${ }^{32}$ im Besitz gültiger Ausweisdokumente beschränkt, was im Vergleich zu 1994 die Zahl der Wähler auf etwa 16 Millionen senkte; dies entsprach 68 Prozent der Wahlberechtigten. Der ANC gewann weitere vier Prozentpunkte hinzu. Mandela trat als Präsident ab, sein Nachfolger wurde Thabo Mbeki. 2004 nahm sich das Ergebnis des ANC mit 69,6 Prozent noch einmal geradezu als Erdrutsch aus. Die DA, aus einer Fusion der Democratic Party (DP) mit der New National Party und der Federal Alliance entstanden, verbesserte die von der DP im Jahr 1999 erzielten Ergebnisse und festigte ihre Position als offizielle Opposition zum ANC. Auf regionaler Ebene regierte der ANC erstmalig in allen neun Provinzen. ${ }^{33}$

Spannung und Erwartungen vor der Wahl 2009 erinnerten ein wenig an die auf die „Befreiungswahlen“ hinführende Periode 1994. Grund dafür war die Aussicht auf Veränderung, sowohl in der Person des beliebten Präsidentschaftskandidaten Jacob Zuma im Ge-

30 Vgl. IPSOS Markinor Press Release vom 3. April 2009.

31 Obwohl die südafrikanische Verfassung ein Zweikammerparlament vorschreibt, assoziieren die meisten die Nationalversammlung mit dem „Parlament“. Die Zweite Kammer ist der ehemalige Senat, der 1996 zum Nationalrat der Provinzen degradiert wurde (90 Sitze). Seine Rolle ist auf die Vertretung der Interessen der Provinzen auf nationaler Ebene beschränkt. Die schwache Position des Rates der Provinzen ist Ausdruck der schwachen föderalen Ausprägung der südafrikanischen Verfassung.

32 Südafrikanische Einwohner, die Staatsbürger anderer Länder und hauptsächlich weiß sind, wurden von den Wahlen ausgeschlossen.

33 Vgl. Electoral Commission of South Africa, http://www.elections.org.za/NPEPWStaticReports/ Default.aspx (Abruf am 25. Mai 2010). 
gensatz zum allgemein unbeliebten $M b e k i$ als auch hinsichtlich der als entwicklungsfähig erscheinenden neuen Wahlalternative zum ANC, COPE. Doch die Erwartungen wurden enttäuscht: Es kam zu keinem nennenswerten Re-Alignment, denn COPE scheint schon wieder am Ende angelangt zu sein. ${ }^{34}$ Zudem werden Südafrikaner aller Gesellschaftsschichten und Lebenslagen immer kritischer gegenüber ihrem scheinbar wohlmeinenden, doch unentschlossenen und moralisch fragwürdigen Präsidenten. ${ }^{35}$

\section{Chancen für eine Mehrparteiendemokratie?}

Trotz des deutlichen Wahlsiegs im April 2009 gelobte der ANC-Vorsitzende und neue Präsident Südafrikas, Jacob Zuma, bei seiner Amtseinsetzung, die Kluft zwischen der regierenden Partei und ihren Konkurrenten zu überbrücken. Er betonte seine Absicht, im Interesse der Nation ein neues Kapitel in den Beziehungen zwischen Regierung und Opposition aufzuschlagen. Doch selbst mit guten Absichten ist in einem von ANC-Abgeordneten und ihren Koalitionspartnern überwältigend dominierten Parlament nicht viel Raum für eine oppositionelle Stimme, die zählt. Dies bestätigt sich besonders unter Bedingungen von Vetternwirtschaft innerhalb des ganzen Staatsapparates. Die Unterscheidung zwischen Partei und Staat, unerlässlich für das ordnungsgemäße Funktionieren einer Demokratie, ist inzwischen gefährlich verwischt. Nicht die Verfassung, die das oberste Gesetz sein sollte, ist die wahre Quelle der Staatsmacht in Südafrika, sondern die ANC-Regierung. Und diese Regierung ist in den letzten Jahren weder auf die Bedürfnisse und Erwartungen der Bürger eingegangen, noch wurde sie vom Parlament zur Rechenschaft gezogen, wie es die Verfassung vorschreibt. Die Art und Weise, wie Mbeki mit dem Parlament bei der Untersuchung der Korruptionsvorwürfe beim Waffenhandel umging und wie seine Regierung generell die öffentliche Diskussion unterdrückte, sorgten dafür, dass der Präsident und seine engen Mitarbeiter ihre eigene Agenda formulieren und verfolgen konnten. Angeblich geschah dies im Namen der „Massen“, deren Los sich ironischerweise nicht viel besserte und deren Unruhe wächst. Die Elite oder der so genannte Native Club brandmarkte alle linken Stimmen als „ultra“ und betrachtete sie als irrelevant; sie bezeichneten Kritiker aus den eigenen Reihen als „gegenrevolutionär“ und werteten deren Meinungen ab; und sie hatten nichts als Geringschätzung übrig für diejenigen, die sie „Kokosnüsse“ (außen dunkel, aber innen weiß) nannten, also schwarze Afrikaner, die die Oppositionsparteien unterstützten. ${ }^{36}$

Mit der Wahl 2009 hielt sich zwar der ANC an der Macht und die erhoffte Umgestaltung der politischen Landschaft in Südafrika blieb aus; sie brachte aber auch ein Potential für Veränderung mit sich, indem sie half, den demokratischen Raum für Debatten in der Öffentlichkeit und im Parlament wieder zu öffnen. Das Parlament scheint die Gelegenheit ergriffen und seine Stimme wieder gefunden zu haben: Die Zuma-Regierung wurde kürz-

34 Ein am 14. Mai 2010 durch das South African Institute of Race Relations veröffentlichter Bericht kam zu dem Schluss, dass das Ausmaß interner Machtkämpfe auf Führungsebene dem Selbstmord von COPE gleichkommt und bedeutet, dass Südafrika wieder zu einem Zwei-ParteienWettbewerb zwischen ANC und DA zurückkehren wird.

35 Sthembiso Msomi, It's Time for Zuma to Be His Own Man, in: Sunday Times vom 9. Mai 2010.

36 Ndebele Njabulo, Acts of Transgression, in: ders., Fine Lines From the Box: Further Thoughts About Our Country, Kapstadt 2004. 
lich durch die Präsidenten beider Kammern, der Nationalversammlung und des Nationalrats der Provinzen, für die Nichtbeantwortung von Fragen gerügt, und mehrere Abgeordnete aller Fraktionen kritisierten das Kabinett, dass es parlamentarische Fragen nicht ordentlich beantwortet habe und sich dem Parlament gegenüber respektlos verhalte. ${ }^{37}$

Gleichzeitig dauern die Spannungen innerhalb der Dreier-Allianz trotz des Weggangs Mbekis an. Daher liegt der Schluss nahe, dass diese dominante, doch nach dem Geist der Verfassung fragwürdige Koalition wahrscheinlich eher früher als später auseinanderfallen wird. Sie hat ihre Legitimation nicht durch das Parlament erhalten, sondern ist das Ergebnis des Befreiungskampfes und der seinerzeit entstandenen Bündnisse. Diese Einheit ist aber längst vergangen und dürfte nicht mehr genügend Bindungskraft entfalten, um das viel zu breite ideologische Spektrum zusammenzuhalten. Ein Auseinanderbrechen würde zu einer Neuordnung der im Land aktiven politischen Kräfte führen und könnte Südafrika vielleicht doch zu einer liberalen Mehrparteiendemokratie zurückführen, wo ein inklusives Programm anstelle eines exklusiven Rassenbildes der entscheidende Faktor im politischen Wettbewerb sein würde.

37 Vgl. Wyndham Hartley, Zuma's Cabinet Told to Answer Questions, in: Business Day vom 12. Mai 2010. 